\title{
Predictive Values of Serum Uric Acid, Mean Platelet Volume and Plateletcrit on Benign Paroxysmal Positional Vertigo
}

\author{
(D) Togay Evrin, (D) Burak Katipoğlu \\ Department of Emergency Medicine, Ufuk University Faculty of Medicine, Ankara, Turkey
}

\begin{abstract}
Aim: There are conflicting study results on correlation of serum uric acid (SUA) with benign paroxysmal positional vertigo (BPPV). In this study, it was aimed to evaluate predictive values of SUA, mean platelet volume (MPV) and plateletcrit (PTC) on BPPV.

Materials and Methods: After the approval of the ethics committee, the files of all patients with BPPV who were admitted to the Emergency Department of Ufuk University between 01.01.2015 and 01.01.2016 were examined retrospectively. White blood cell, MPV, PTC and SUA levels of patients were evaluated.

Results: There was no statistically significant difference between the groups in terms of gender distribution $(p>0.05)$. The mean age of the control group ( $31.25 \pm 11.46$ years) was lower than the vertigo group (44.71 \pm 19.38 years), but this difference was not statistically significant $(p>0.05)$. The difference between two groups in terms of MPV, PTC and SUA parameters was statistically significant $(p<0.05)$. The sensitivity was found to be $92.1 \%$ and the specificity was $47.8 \%$. For a PTC value of $0.224 \%$, the sensitivity was $23.7 \%$ and the specificity was $87 \%$. For SUA, area under curve was $71.6 \%$ indicating that SUA had a predictive value for peripheral vertigo $(p<0.01)$. The sensitivity of SUA was $55.3 \%$ and the specificity was $21.7 \%$ for a SUA value of 4.1. The sensitivity of SUA was 15.8 and the specificity was $87 \%$ for a SUA value of 6.55 .

Conclusion: Analysis results were in accordance with volume ure in which SUA is suspected in idiopathic BPPV. In addition, PTC was also correlated with BPPV.
\end{abstract}

Keywords: Serum uric acid, mean platelet volume, plateletcrit, benign paroxysmal positional vertigo

\section{Introduction}

Benign paroxysmal positional vertigo (BPPV) is a common healthcare problem in the elderly. BPPV is characterized by short vertigo episodes with movement of the head (1). BPPV has a high incidence rate, approximately $17-20 \%$ of peripheral vertigo (2). Most BPPV cases are idiopathic. However, some risk factors such as ear surgery, labyrinthitis, vestibular neuritis, and head trauma are common causes for BPPV. In addition, the symptoms and prevalence increase with age (3). The common diagnostic procedure includes visualization of nystagmus and medical history of the patient (4). The prevalence of BPPV is reported as $25 \%$ in older people over 70 years with complaints of more than one year (5). More than 90\% of BPPV patients can be treated with maneuvers. Most patients with vertigo state that closing the eyes reduces the symptoms (6). Current treatments include manual repositioning procedures such as Gufoni, Semont, Epley or Barbecue procedures (2).

Serum uric acid (SUA) is a purine metabolism product. Some studies have shown that SUA may be an important biomarker for specific motor features (7). Its association with diabetic peripheral neuropathy (8), cardiovascular diseases $(9,10)$, ischemic stroke (11) and many other clinical syndromes was reported. Similar to SUA, mean platelet volume (MPV) and plateletcrit (PTC) have been reported as biomarkers for some health problems. 
In this study, it was aimed to evaluate predictive values of SUA, MPV and PTC in BPPV patients who applied to our emergency clinic.

\section{Materials and Methods}

Patient files of all patients who were admitted to the emergency department of Ufuk University with BPPV between 01.01.2015 and 01.01.2016 were examined retrospectively. White blood cell (WBC), MPV, platelet distribution width (PDW) and SUA values of patients were evaluated. This study was conducted in accordance with World Medical Association Declaration of Helsinki "Ethical Principles for Medical Research Involving Human Subjects", (amended in October 2013). No consent form was obtained from the patients due to retrospective nature of the study. This study was approved by the Ethics Committee of Ufuk University with a number of 26022016-2 and a date of 26.02.2016.

\section{Statistical Analysis}

Nominal and binary parameters were described with frequency analysis. The values for research parameters were described as mean \pm standard deviation. Chi-square analysis was used for nonparametric nominal parameter differences. Kolmogorov-Smirnov test was used to examine the normality of scale parameters. Since all parameters showed non-normal distribution, Mann-Whitney $U$ test was used to define differences between control and vertigo groups. ROC Curve analysis was used to define sensitivity and specificity of the parameters. All analyzes were performed using SPSS 17.0 for Windows (Chicago, Illinois) at 95\%-99\% Cl levels.

\section{Results}

The results of gender and age distribution and difference analysis for patient groups are given in Table 1.

Fifty-two point two percent of the subjects in the control group were female and $47.8 \%$ were male. There were 26 women (68.4\%) and 12 men (31.6\%) in the vertigo group. The difference between the groups in terms of gender distribution was not statistically significant $(p>0.05)$. The mean age of the control group (31.25 \pm 11.46 years) was lower than the vertigo group (44.71 \pm 19.38 years), but this difference was not statistically

Table 1. The gender and age distribution of groups

\begin{tabular}{|c|c|c|c|}
\hline & Control $(n=24)$ & Vertigo $(n=38)$ & p \\
\hline \multicolumn{3}{|l|}{ Gender } & \multirow{3}{*}{$0.204^{a}$} \\
\hline Female, n (\%) & $12(52.2)$ & $26(68.4)$ & \\
\hline Male, $\mathrm{n}(\%)$ & $11(47.8)$ & $12(31.6)$ & \\
\hline Age, Mean \pm SD & $31.25 \pm 11.46$ & $44.71 \pm 19.38$ & $0.074^{b}$ \\
\hline
\end{tabular}

significant $(p>0.05)$. The results of some blood parameters in the control and vertigo groups and difference analysis are given in Table 2.

WBC, MPV, PDW and SUA levels of the control group were higher than the vertigo group. PTC level of the vertigo group was higher than the control group. The difference between two groups in terms of MPV, PTC and SUA parameters were statistically significant $(p<0.05)$. The results of the ROC analysis for examining the predictive values of the significant parameters on vertigo are given in the Figure 1.

The area under the curve was found to be 0.969 . The predictive power of PTC was found to be $69.6 \%$. The results of ROC analysis were statistically significant ( $p>0.05)$. For a PTC value of 0.1565 , the sensitivity was $92.1 \%$ and the specificity was $47.8 \%$. For a PTC value of $0.224 \%$, the sensitivity was $23.7 \%$ and the specificity was $87 \%$. For SUA, the area under the curve was $71.6 \%$ indicating that uric acid had a predictive value for peripheral vertigo $(p<0.01)$. The sensitivity of SUA was $55.3 \%$ and the specificity was $21.7 \%$ for a SUA value of 4.1. The sensitivity of SUA was $15.8 \%$ and the specificity was $87 \%$ for a SUA value of 6.55 . The results of univariate and multiple regression analysis are given in the Table 3.

Univariate and multiple regression analysis results showed that only SUA $(p<0.01)$ and MPV $(p<0.05)$ had a predictive value for

Table 2. The blood parameters and comparison of the control and vertigo groups

\begin{tabular}{|c|c|c|c|}
\hline Parameters $^{\mathrm{a}}$ & Control $(n=24)$ & Vertigo $(n=38)$ & $p^{b}$ \\
\hline WBC & $7.61 \pm 1.41$ & $7.55 \pm 2.65$ & 0.240 \\
\hline MPV & $8.20 \pm 2.13$ & $7.23 \pm 1.40$ & 0.036 \\
\hline PDW & $17.84 \pm 3.60$ & $17.30 \pm 3.26$ & 0.829 \\
\hline PTC & $0.19 \pm 0.10$ & $0.21 \pm 0.07$ & 0.011 \\
\hline Uric acid & $5.39 \pm 1.19$ & $4.45 \pm 1.43$ & 0.005 \\
\hline
\end{tabular}

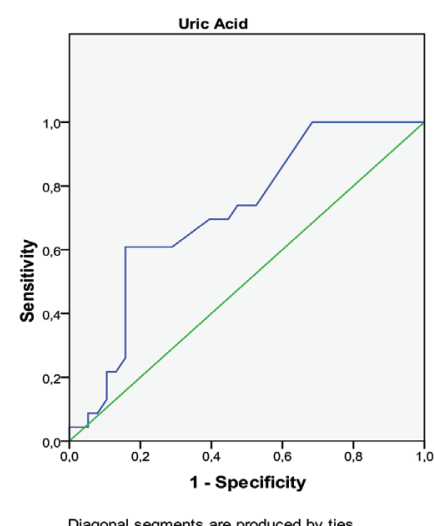

Figure 1. The results of the ROC analysis to examine the predictive value of significant parameters on vertigo 
Table 3. Univariate and multiple regression analysis results

\begin{tabular}{|c|c|c|c|c|}
\hline \multirow{2}{*}{ Variables } & \multicolumn{2}{|l|}{ Univariate } & \multicolumn{2}{|l|}{ Multiple } \\
\hline & OR $(95 \% \mathrm{Cl})$ & $\mathbf{p}$ & OR $(95 \% \mathrm{Cl})$ & $\mathbf{p}$ \\
\hline Gender & $2.00(0.818-4.89)$ & 0.129 & - & - \\
\hline WBC & $1.042(0.862-1.259)$ & 0.674 & - & - \\
\hline MPV & $0.738(0.555-0.981)$ & 0.036 & $0.668(0.474-0.942)$ & 0.022 \\
\hline PDW & 0.984 (0.857-1.130) & 0.818 & - & - \\
\hline Uric acid & $0.614(0.425-0.886)$ & 0.009 & $0.592(0.399-0.880)$ & 0.01 \\
\hline
\end{tabular}

single parameter level, whereas MPV and SUA had predictive value at multiple parameter levels. These results showed that SUA had a predictive value for vertigo.

\section{Discussion}

Vertigo is one of the most important and common health problems in emergency services. In addition, vertigo significantly reduces the quality of life in patients. Thus, there have been researches on the diagnosis and treatment of vertigo. In emergency services, its diagnosis is more important and researches on this area focus on diagnosis in order to prevent misuse of medical agents. The SRM-IV vestibular function diagnosis and treatment procedure helps in the diagnosis and treatment of BPPV (2). Moreover, oxidative stress has been reported to induce pathogenesis of BPPV (12). Thus, it can be argued that BPPV has a complex diagnostic procedure and risk factors. Some researchers reported that vertigo has a significant correlation with age, BMI and gender (13). In our study, gender and age distributions of patient groups were not statistically significant. This shows that the study cohort does not include age and gender effects on vertigo and this increases the reliability of the analysis results.

In the literature, researches on the relationship between BPPV and SUA can be classified into two groups. In the first group, researches argue that SUA is correlated with BPPV. In the second group, researches argue that BPPV patients have lower SUA levels, but the correlation is insignificant. Celikbilek et al. (14) reported that SUA level was positively correlated with BPPV. Similarly, Chang et al. (15) reported a relationship between vertigo and SUA. Yuan et al. (16) reported that SUA was lower in BPPV patients, but the difference was not statistically significant. In another research conducted by Yuan et al. (1), SUA levels of BPPV patients were found to be lower with an insignificant risk factor for BPPV. Jeong and Kim (17) reported that SUA was not a risk factor for idiopathic BPPV. In our study, PTC levels were lower in the vertigo group. The other blood parameters (WBC, MPV and
PDW) were higher in the control group. Thus, it is recommended to evaluate SUA with MPV at multiple variables level.

\section{Conclusion}

In literature, there are studies arguing whether SUA is a risk factor for BPPV. In our study, analysis results are in accordance with the literature suggesting that SUA predicts idiopathic BPPV. In addition, PTC was also correlated with BPPV. On the other hand, both literature research groups with significant or insignificant correlation stated that SUA levels were lower in the BPPV patients. In this respect, it can be argued that the increasing number of researches and meta-analysis in this issue, and the increasing number of patient population may change the direction of the literature towards the significant correlation results. For emergency clinics, SUA for BPPV patients may help physician to predict or suspect for BPPV.

\section{Ethics}

Ethics Committee Approval: The research has been approved by the Ufuk University Ethics Committee on 26.02.2016 and has therefore been performed in accordance with the ethical standards laid down in the 1964 Declaration of Helsinki and its later amendments (approval number: 26022016-2).

Informed Consent: No consent form was obtained from the patients due to retrospective nature of the study.

Peer-review: Externally and internally peer-reviewed.

\section{Authorship Contributions}

Surgical and Medical Practices: B.K., Concept: T.E., Design: T.E., Data Collection or Processing: B.K., Analysis or Interpretation: T.E., Literature Search: T.E., Writing: B.K.

Conflict of Interest: No conflict of interest was declared by the authors.

Financial Disclosure: The authors declared that this study received no financial support.

\section{References}

1. Yuan J, Chen Y, Chen Y, Niu S, Li S, Dong Q, et al. Relationship between serum level of uric acid and benign paroxysmal positional vertigo. Zhonghua Yi Xue Za Zhi. 20153;95:344-8.

2. Wang $\mathrm{N}$, Zhou H, Huang H, Geng D, Yang X, Yu C. Efficacy of SRM-IV Vestibular Function Diagnosis and Treatment System in Treating Benign Paroxysmal Positional Vertigo. Iran J Public Health. 2018;47:641-7.

3. Saeed BMN, Omari AF. Climatic variations and benign paroxysmal positional vertigo. J Otol. 2016;11:33-7.

4. Ferreira MM, Ganança MM, Caovilla HH. Subjective visual vertical after treatment of benign paroxysmal positional vertigo. Braz J Otorhinolaryngol. 2017;83:659-64.

5. Ribeiro KF, Oliveira BS, Freitas RV, Ferreira LM, Deshpande N, Guerra RO. Effectiveness of Otolith Repositioning Maneuvers and Vestibular 
Rehabilitation exercises in elderly people with Benign Paroxysmal Positional Vertigo: a systematic review. Braz J Otorhinolaryngol. 2018;84:109-18.

6. Chin S. Visual vertigo: Vertigo of oculomotor origin. Med Hypotheses. 2018;116:84-95.

7. Huang X, Ng SY, Chia NS, Acharyya S, Setiawan F, Lu ZH, et al. Serum uric acid level and its association with motor subtypes and non-motor symptoms in early Parkinson's disease: PALS study. Parkinsonism Relat Disord. 2018;55:50-

8. Lin X, Xu L, Zhao D, Luo Z, Pan S. Correlation between serum uric acid and diabetic peripheral neuropathy in T2DM patients. J Neurol Sci. 2018;385:7882.

9. Wu AH, Gladden JD, Ahmed M, Ahmed A, Flippatos G. Relation of serum uric acid to cardiovascular disease. Int J Cardiol. 2016;213:4-7.

10. Jun JE, Lee YB, Lee SE, Ahn JY, Kim G, Jin SM, et al. Relation of serum uric acid to cardiovascular disease. Atherosclerosis. 2018;272:233-9.

11. Bayar N, Küçükseymen S, Güven R, Erkal Z, Köklü E, Yüksel iÖ, et al, Association between serum uric acid and ischemic stroke in patients with non valvular paroxysmal atrial fibrillation. Int J Cardiovascular Academy. 2017;3:118-21.
12. Güçlütürk MT, Ünal ZN, İsmi $O$, Çimen MB, Ünal M. The Role of Oxidative Stress and Inflammatory Mediators in Benign Paroxysmal Positional Vertigo. J Int Adv Otol. 2016;12:101-5.

13. Filippopulos FM, Albers L, Straube A, Gerstl L, Blum B, Langhagen T, et al. Vertigo and dizziness in adolescents: Risk factors and their population attributable risk. PLoS One. 2017;12:e0187819.

14. Celikbilek A, Gencer ZK, Saydam L, Zararsiz G, Tanik N, Ozkiris M. Serum uric acid levels correlate with benign paroxysmal positional vertigo. Eur J Neurol. 2014;21:79-85.

15. Chang CC, Chang WN, Huang CR, Liou CW, Lin TK, Lu CH. The Relationship between Isolated Dizziness/Vertigo and the Risk Factors of Ischemic Stroke: A Case Control Study. Acta Neurol Taiwan. 2011;20:101-6.

16. Yuan J, Dai J, Li WA, Hu W. Factors Associated with Benign Paroxysmal Positional Vertigo: A Chinese Case-Control Study. Med Sci Monit. 2017;23:3885-9.

17. Jeong SH, Kim JS. The Effect of Serum Uric Acid in Generating Idiopathic Benign Paroxysmal Positional Vertigo. Research in Vestibular Science. 2010;9:27-31 